\title{
Detección precoz de hipotiroidismo congénito
}

\author{
Dra María E. Wilshaw Z. ${ }^{\text {; }}$ Dr. Carlos Viliarroel F. ${ }^{2}$; B.Q. Gina Parodi C. ${ }^{2}$; \\ Dr. Roger Lamas C. ${ }^{3}$; T.M. Haydée Valdés $G{ }^{2}$
}

Neonatal screening of congenital hypothyroidism

Thity two (1\%) of 3084 screened newborn infants had high TSH levels. All were recalled for confirmation and in anly onc of them congenital hypothyroidism was confirmed: he had no clinical evidence of the disease, but low normal $\mathrm{T}_{4}$, high $\mathrm{TSH}$ plasma levels and an ectopic sublingual thyroid. detected by $\mathrm{T}_{\mathrm{c}} 99_{\mathrm{m}}$ scan, were demonstrated. (Key words: Hy pothyroidism, congenital, TSH screening).

La identifjcación precoz de los recién nacidos con hipotiroidismo congénito, sigue siendo una theta en el campo pediátrico y endocrinológico. Aunque el hipotiroidismo congénito es la afección metabólica más frecuente del recjén nacido ${ }^{2}$, su diagnóstico clínico es dificil por la baja frecuencia e inespecificidad de los síntomas que presenta el neonatol. De las experiencias de Brasel y Boyd sobre influencia de las hormonas tiroídeas en el desarrollo y metabolismo dei sistema nervioso central y de la observación de pacientes con déficit de hormonas tiroídeas no tratados en los primeros meses de vida, se puede concluir que las consecuencias más severas de esta enfermedad ocurren en el sistema nervioso central y pueden ser evitadas con tratamiento oportuno ${ }^{2-5}$.

Las limitaciones del examen clínico en las primeras semanas de vida ${ }^{6}$ y la importancia del tratamiento precoz ${ }^{3,4}$ han incentivado la búsqueda de métodos de laboratorio que permitan detectar recién nacidos apartitemente normales pero metabólicamente enfermos y hechó surgir programas de pesquisa neonatal para el hipotiroidismo congénito ${ }^{7}$ adosados a los previamente existentes para fenilquetonuria, primero en Canadá en el año 1974, posteriormente en EE.UU., varios países de Europa, Japón y en Sudamérica, donde fue adoptado por Argentina desde 1980. Las primeras experiencias preconizadas $^{9,9}$ utilizaron la medición de tiroxina (T4), en una gota de sangre obtenida por punción de

\footnotetext{
k. Servicio de pediatría, sección endocrinología, Hospizal Militar.

2. Servicio de medicina nuclear, Hospital Militar.

3. Servicio de neona tología, Hospital Militar.
}

talón, entre el tercer y sexto día de vida, o sangre de cordón umbilical10.11 y también se emplearon mediciones de TSH en gota de sangre, en papel filtro, por punción de talón $12,13,14$. Con el estudio de TSH se pesquisan $90 \%$ de los hipotiroidismos debidos a fallas tiroídeas prima. rias, pero no se descartan las fallas hipotálamohipofisiarias, sin embargo, algunos paises ${ }^{15} \mathrm{em}$. plean el método en sus planes nacionales, por ofrecer una baja proporción de rellamados: desde que se incorporaron estos programas en Suiza, la frecuencia de detección ha sido de $1 / 3000$ recién nacidos y solamente $5 \%$ de los pacientes detectados presentaban signos clínicos sugerentes de la enfermedad. Desde, noviembre de 1983 , se ha aplicado en la maternidad de nuestro hospital, un programa de pesquisa con TSH en todos los recién nacidos cuyos resultados se exponen a continuación con el objeto de informar sobre los resultados.

\section{MATERIAL Y METODO}

Se obtuvieron muestras de sange por punción de talón en 3.084 recién nacidos, desde noviembre de 1983 hasta febrero de este año en ta maternidad del.Hospital Militar de Santiago de Chile. Las muestras se recolectaron en tarjetas de papel filtro S\&S 903, proporcionada por dos marcas de reactivos comerciales de radioinmunoanálisis para TSH y en $90 \%$ de los casos fueron obtenidas a las 72 horas de vida (rango 24 a 140 horas). Las tarjetas con las muestras fueron secadas individualmente a temperatura ambiente por 2 horas y enviadas al laboratorio de medicina nuclear donde se almacenaron a $4^{\circ} \mathrm{C}$ de temperatura hasta su procesamiento semanal. Para el procesamiento, se cortaron, de las tarjetas, discos de 4,5 a $7 \mathrm{~mm}$ de diámetro, mediante un perforador especial proporcionado en cada equipo de reactivos. El volumen de suero contenido en cada disco era de aproximadamente $5 \mu$, pudiendo llegar hasta $10 \mu$, 
caso en el que es necesario hacer la correccion respectiva conforme a las indiççiones del fabricante.

El procesamiento de las muestras se realizó mediante radioinmunoanálisis de doble anticuerpo, que se basa en la competencia, por los sitios de unión de un anticuerpo específico, entre el TSH marcado con Iodo-125 y el TSH contenido en la muéstra de papel filtro. Después de una serie de incubaciones durante 48 horas en lemperatura ambiente se agrega el segundo anticuerpo сол una solución precipitante de polientilenglicol (PFG) y por centrifugación se separan las distintas fases. El TSH libre, que queda en el sobrenadante, es aspirado y la radiactividad residual, unida al segundo anticuerpo, es medida en contador de radiación gamma. El cálculo de las concentraciones de TSH en las muestras se tealizó mediante técnicas habitualeg de radioinmunoanálisis $16,17,18$.

\section{RESULTADOS}

De 3.084 determinaciones, 1.100 fueron hechas con uno de los tipos de reactivos de radioinmunoanálisis y las restantes con otro. En las primeras 1.100 se fijó el valor de corte de normalidad en $25 \mu \mathrm{UI} \times \mathrm{ml}$, resultando $99 \%$ de los valores de las concentraciones de TSH iguales o menores a esta cifta y sólo $1 \%$ de los recién nacidos (11 casos) fueron vueltos a llamar. En los 1.984 en que se empleó la segunda marca de reactivos se fijó el valor de corte de normalidad en $30 \mu \mathrm{UI} \times \mathrm{ml}$, resultando 1.963 determinaciones (99\%) con concentraciones de TSH jguales 0 menores a esta cifra y 21 ( $1 \%$ ) mayores (Figura l). En síntesis, 32 pacientes debieron ser llamados ${ }^{15}$, para realizar examen clinico y mediciones plasmaticas de triyodotironina, tiroxina y TSH, y evaluación de la edad ósea mediante radiografías de rodilla, resuitando su concentración promedio de TSH de 9,32 $\mu \mathrm{UI} \times \mathrm{ml}$ con \pm 7,29 (D.E.) y, sólo en uno se encontraron cifras anormales de TSH plasmático (más de $200 \mu \mathrm{Ul} x$ ml) con $\mathrm{T} 3$ normal (125 $\mathrm{ng} \times \mathrm{ml} \times \mathrm{x} 4 \mathrm{en}$ limite normal bajo $(7,2 \mu \mathrm{g} \times \mathrm{dl})^{10}$ : su estudio mediante cintigrama tiroideo con $\mathrm{T}_{\mathrm{c}}-99 \mathrm{n}$ comprobó la existencia de una glándula tiroides ectópica sublingual sin alteraciones clínicas visibles. No se observaron diferencias en las concentraciones de TSH obtenidas con respecto al momento en que se tomaron las muestras en los pacientes de esta serie.

\section{DISCUSION}

Entre estos 3.084 recién nacidos sometidos a pesquisa de hipotiroidismo congénito por medición de TSH neonatal, $1 \%$ (32 niños) tenían valores superiores al establecido como limite máximo normal, constituyendo el grupo de rella. mado, proporción similar a la observada en otros

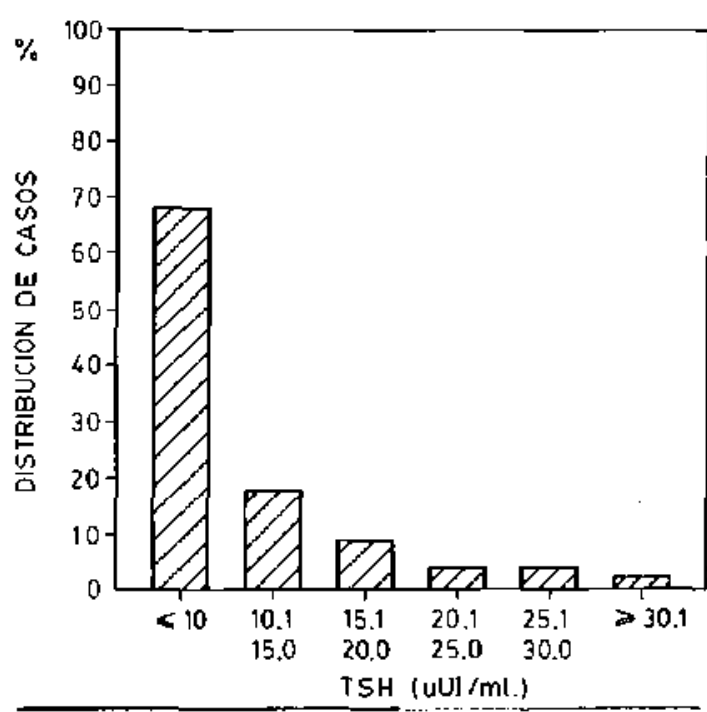

Figura 1. Distribución porcentual de los iasos de acuerdo con la concentración plasmática de TSH.

programas que utilizan el mismo método y cuya casuistica supera la nuestra ${ }^{15}$.

El único paciente entre los 32 rellamados, tenia TSH sérico aumentado $(<200 \mu \mathrm{UI} \times \mathrm{ml})$, pero su T4 estaba en el límite normal bajo, era portador de un tiroides ectópico sublingual detectado en el cintigrama con $\mathrm{Tc}^{99} \mathrm{~m}$ y no tenía signos clínicos que permitieran el diagnóstico: enfatizamos la importancia del TSH, justamente en este caso donde el T4 estaba en el rango normal.

E) TSH constituye el mejor indicador de hipotiroidismo primario cumpliendo así el objetivo de los programas de detección preconizados por los paises reunidos en la II Conferencia Internacional realizada en Tokio en 1982. Tiene como limitaciones que no permite detectar las fallas hipotálamo hipofisiarias y que en los prematuros los valores de TSH en los primeros días de vida serían más bajos por inmadurez de su eje hipotálamo-hipofisiario-tiroídeo ${ }^{19}$.

La factibilidad de la técnica por punción de talón, la facjlidad de su envio en tarjeta y la posibilidad de tomar la muestra antes de las 72 horas y pasadas las $\mathbf{2 4}$ horas de vida, constituyen hechos importantes para formular un programa masivo. Además debe considerarse el bajo porcentaje (1\%) de casos de rellamado, lo que interesa en e] costo de un programa de este tipo.

De acuerdo a las publicaciones que relacionan el pronóstico del coeficiente intelectual con la oportunidad del tratamiento, estaríamos ofreciendo a este paciente las mejores condiciones

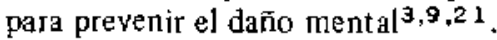




\section{RESUMEN}

Se presenta el primer programa de detección precoz de hipotiroidismo congénito en nuestro paj́s, realizado desde 1983 en la Maternidad del Hospital Militar de Santiago.

De 3.084 recién nacidos tenemos 32 con niveles de TSH superiores al valor de corte y que ingresaron a un programa de rellamado, lo que representa un $1 \%$ del total. El único caso confirmado corresponde al mayor valor de TSH (más de $200 \mu$ UI $\mathrm{x} \mathrm{ml}$ ) y que en la reevaluación no presenta signos clínicos de sospecha, pero cuyo diagnóstico se confirma por niveles límites normales bajo de $\mathrm{T} 4 \mathrm{y}$ altos de $\mathrm{TSH}$ en el plasma. El cintigrama confirma un tiroides ectópico (sublingual).

Se enfatiza la importancia de disponer de un plan nacional de detección precoz de hipotirojdismo congénito para lo cual puede servir de base nuestra experiencia y resultados.

\section{REFERENCIAS}

I. Fisher, D.: Detección de Hipotiroidismo en neonatos. Clin. Pediatr. Am. 3: 423, 1978.

2. Brasel, J., Boyd, D.: Influence of thy roid hormone on fetal brain growth and development in: Fisher $A$. and Burrow G. (Ed.) Perinatal thyroid psysiology and disease, New York, Raven Press, 1975, pp. $59-71$.

3. Klein, A., Mettzer, S., Kemmy, F.: Improved prognosis in Congenital Hypothyroidism Treated before age three montlis. J. Pediatr. 81: $912,1972$.

4. New England Congenital Hyporhyroidism Collabo. rative. Effects of Neonatal Screening for Hypothyroidism: Prevention of Mental Retardation by treatment before clinical manifestation. Lancet 2 : 1095,1981

5. Glorieux, J., Dussauti. J., Letarte, H. et al: Prelịninary results on the mental development of hypothyroid infants detected by Quebec Screening Program. J. Podiatr. 102; 19, 1983.
6. Jacobsen, B., Brandt, N.: Congenital Hy pothyroidism in Denmark. Arch. Dis. Child. 56: 134, 1981.

7. Dussault, J., Coulombe, C., Laberge, C. et al: Preliminary report on a mass screening program for reonatal hypothyroidism. J. Pediats, 86:670, 1975.

B. Dussenit, J., Letorte, H., Guyda, H., et al: Thyroid funtion in neonatal hypothyroidism. 3. Pediatr. 89: $54 \mathrm{~L}, 1976$.

9. Dussoult, J., Parlow, A., Letorte, J., at al: TSH measurements from blood spots on filter paper: $A$ confirmatory screening test for neonatal hypothyroidistr. J. Pediatr. 89: 550, 1976.

10. Klein, $A$, Foley, $T$, Larsen, $R,:$ Neonatal thyroid funtion in congenital hypothyroidism. J. Pediatr. 89: $545,1976$.

11. Wolfish, P.: Screening for neonatal hypothyroidism using cord blood T4 measurements: Comparation to neonatal capillary dried blood and serum T4 screening methods In Fisher D.A. and Burrow G.N. (Ed) Perinatal thyroid physiology and disease New York, Raven Press, 1975. pp 263-269.

12. Dussaut, J., Morissette, J., Letorte, J. et al: Modification of a screening program for neonatal hypotluyroidism. J. Pediatr. 92: 274, 1978.

13. Foley, $T$. Klein, $A$.: Adaptation of TSH filter paper method for regionalized screening for congenital hy pothyroidism. J. Lab. Clin. Med. 90: 11, 1977.

14. Minoru. I., Enomoto, K.: Measurement of thyroid stimulating hormone in dried blood spot. Lancet Dec. 20: 1233, 1975.

15. Ilig, R.: Congenital Hypothyroidism. Clin. Endocrinol Metab. 8: 49, 1979.

16. Kenneth, $F_{\text {. }}$ : Fnd point parameter adjustment on a small desktop programable calculator for logic analysis of radioimmunoassay data, Clin. Chem. 22: $1383,1976$.

17. Rodbord, D.: Rapid calculation of radioimmunoassay results. J. Lab. Clin. Med. 74: 770, 1969.

18. Burger, $H$. . A generalized computer program for the treatment of data from competitive proteinbinding assay including radioimmunoassays. J. Lab. Clin, Med. 80: 302, 1972.

19. Fisher, D.: Second International Conference on Neonatal Thyroid Screening: Progress report. J. Pediatr. 102: 653, 1983.

20. Alem. J., Larsson, A, Zelterström, R.: Congenita1 hypothyroidism in Sweden. Acta Paediatr. Scand. 70: $907,1981$.

21. Kaplan, M. Kauli, R., Lubin, T.: Ectopic thyroid gland. J. Pediatr. 92: 205, 1978. 